\title{
Wide localized solitons in systems with time- and space-modulated nonlinearities
}

\author{
L. E. Arroyo Meza, ${ }^{*}$ A. de Souza Dutra, ${ }^{\dagger}$ and M. B. Hott ${ }^{\ddagger}$ \\ Universidade Estadual Paulista, Campus de Guaratinguetá, Departamento de Física e Química, 12516-410, Guaratinguetá, \\ São Paulo, Brazil
}

(Received 21 May 2012; published 23 August 2012)

\begin{abstract}
In this work we apply point canonical transformations to solve some classes of nonautonomous, nonlinear Schrödinger equations, namely, those which possess specific cubic and quintic (time- and space-dependent) nonlinearities. In this way we generalize some procedures recently published which resort to an ansatz to the wave function and recover a time- and space-independent nonlinear equation which can be solved explicitly. The method applied here allows us to find wide localized (in space) soliton solutions to the nonautonomous, nonlinear Schrödinger equation. We also generalize the external potential which traps the system and the terms of the nonlinearities.
\end{abstract}

DOI: 10.1103/PhysRevE.86.026605

\section{INTRODUCTION}

Investigations into the nonlinear Schrödinger equation (NLSE) [1] have increased in recent decades. The importance of such investigations is not only due the possible real-world applications of NLSE or the Gross-Pitaevskii equation (GPE) [2] but also due to the possibility, on the theoretical side, of increasing the class of nonlinear integrable models [3].

The applications of NLSE and/or GPE with spatially dependent cubic and quintic (CQ) nonlinearities can be appreciated, for example, in pulse propagation in optical fibers [4], in photonic crystals [5], and in the study of Bose-Einstein condensates (BECs) [6], whose nonlinearities are driven by means of optical interactions as well.

Specificly, dark and bright solitons have been observed in various nonlinear physical phenomena. Investigation of bright and dark solitons is useful for understanding the properties of BECs. Bright solitons are characterized by a localized maximum in the density profile without any phase jump across it. In the relevant experiments, this type of soliton is formed by utilizing a Feshbach resonance to change the sign of the scattering length from positive to negative. On the other hand, dark solitons may also be considered as moving domain walls which separate regions of a condensate with different values of the order parameter. In fact, dark solitons are density dips characterized by a phase jump of the wave function at the position of the dip and can be generated by means of phase-engineering techniques [7]. Thus, there are many possibilities for managing these solitons, strongly justifying a quest for novel analytical solutions.

The techniques for managing the nonlinearities have been improved a lot, and in some cases, the nonlinear equations governing the system present not only space- [8] but also timedependent nonlinearities $[9,10]$. In this case one talks about the so-called nonautonomous NLSE [11], whose localized wave solutions, in some specific cases, were found by Serkin and Hasegawa [12] with a similarity transformation, which maps the nonautonomous NLSE onto a nonlinear stationary equation whose solutions are well known.

\footnotetext{
*luisarroyo@feg.unesp.br

†dutra@feg.unesp.br

${ }^{\ddagger}$ marcelo.hott@pq.cnpq.br
}

PACS number(s): 05.45.Yv, 03.75.Lm, 42.65.Tg

A similar procedure has been carried out in a recent study by Beitia, Pérez-García, Vekslerchik, and Konotop (BPVK) [13]. Concretely, the authors relate the nonautonomous NLSE with cubic nolinearity (CNLSE)

$$
i \frac{\partial \Psi}{\partial t}=-\frac{\partial^{2} \Psi}{\partial x^{2}}+v(x, t) \Psi+g(x, t)|\Psi|^{2} \Psi,
$$

and the stationary CNLSE with constant coefficients

$$
\mu \Phi=-\frac{\partial^{2} \Phi}{\partial X^{2}}+G|\Phi|^{2} \Phi
$$

( $\mu$ is the eigenvalue of the nonlinear equation known as chemical potential) by using the ansatz

$$
\Psi(x, t)=\rho(x, t) \exp [i \varphi(x, t)] \Phi[X(x, t)]
$$

for the solution of Eq. (1). The variable $X$, which in Eq. (2) plays the role of space coordinate, is in fact a function $F(\xi)$ of the specific combination $\xi(x, t)=\gamma(t) x+\delta(t)$ of the space coordinate and the time. In such an approach, which provides analytical solutions for the wave function $\Psi(x, t)$, one finds out that specific forms of the trapping potential $v(x, t)$ and the nonlinearity function $g(x, t)$ can be explored and that there is an intrinsic dependence of $v(x, t), g(x, t), \rho(x, t)$, and $\varphi(x, t)$ on $\gamma(t), \delta(t)$, and $F(\xi)$; that is, one may choose conveniently $\gamma(t), \delta(t)$, and $F(\xi)$ to obtain $v(x, t), g(x, t)$, and, consequently nonsingular $\rho(x, t)$ and single-valued phase $\varphi(x, t)$.

More recently, in the work by Avelar, Bazeia, and Cardoso (ABC) [14], the authors followed the BPVK approach but extended the problem, focusing their attention on the nonautonomous cubic and quintic nonlinear Schrödinger equation (CQNLSE). They have also obtained analytic localized solutions of the bright or dark type (breathing, resonant, quasiperiodic, and moving breathing solutions), depending on whether the eigenvalue $\mu$ vanishes.

The nonautonomous CQNLSE is obtained by adding a term $h(x, t)|\Psi|^{4} \Psi$ to the right side of Eq. (2), and the stationary CQNLSE with constant coefficients is given by

$$
\mu \Phi=-\frac{\partial^{2} \Phi}{\partial X^{2}}+G_{3}|\Phi|^{2} \Phi+G_{5}|\Phi|^{4} \Phi .
$$

We have noticed that when the similarity transformation is applied, the trapping potential $v(x, t)$ always has a quadratic term, namely $\omega^{2}(t) x^{2}$, which constitutes a harmonic oscillator 
with time-dependent frequency. The frequency is related to $\gamma(t)$ and its time derivatives and, when $d^{2} \delta / d t^{2} \neq 0$, one finds a driven time-dependent harmonic oscillator whose force is also time dependent. Such kind of problem is consecrated in the literature of the time-dependent Schrödinger equation concerning the analysis of dissipative effects in quantum fluctuations. Moreover, such systems have found real-world applications in quantum optics [15] and plasma physics [16]. Among the approaches applied to solve the Schrödinger equation for the time-dependent (driven) oscillator, one resorts to point canonical transformations on the coordinates and a rescaling of the time in such a way that the problem can be transformed into a Schrödinger equation for the harmonic oscillator with constant frequency [17]. As a matter of fact, in some specific cases, the problem can be even reduced further into a Schrödinger equation for a free particle [18].

One of the main goals of the present work is to apply successfully the same canonical point transformations for solving some time-dependent Schrödinger equations to the problem of the nonautonomous CQNLSE mentioned above. We show that the point canonical transformation followed by an appropriate redefinition of the wave function and additional transformations of variables also leads to the stationary CQNLSE with constant coefficients (4), without resorting to any ansatz on the form of the wave function $\Psi(x, t)$ in Eq. (3).

The approach adopted here is straightforward, and as a result of it one can see clearly the dependence of the trapping potential $v(x, t)$ and the inhomogeneous coefficients $g(x, t)$ and $h(x, t)$ with the functions $\gamma(t), \delta(t)$, and $F(\xi)$. With this approach, the constraints over the functions $\gamma(t), \delta(t)$, and $F(\xi)$ in order to render the wave function $\Psi(x, t)$ well defined are also evident. By following the mapping approach presented in Ref. [19] we present the soliton solutions for Eq. (4) in terms of the Weierstrass elliptic function, such that by considering specific sets of the parameters $\mu, G_{3}, G_{5}$ and an arbitrary constant of integration we not only recover the results found in Refs. [13] and [14] but also present some new soliton solutions by using the set of functions $\gamma(t), \delta(t)$, and $F(\xi)$.

We also deal with extensions of the models by including other trapping potentials which are a mix of circular functions with the time-dependent harmonic oscillator and also comment on a generalization of the nonautonomous time-dependent nonlinear Schrödinger equation by considering a nonpolynomial nonlinearity.

In the next section we present the approach to map the nonautonomous CQNLSE onto a stationary CQNLSE. The third section is devoted to present the solutions of Eq. (4) in terms of the Weierstrass elliptic function where some examples of wide bright and dark solitons are shown. In the fourth section we consider other kinds of trapping potential besides the persistent time-dependent harmonic oscillator and comment on a nonpolynomial nonlinearity. The fifth section is left for the conclusions.

\section{THE APPROACH}

In this section we present the approach by focusing on the nonautonomous nonlinear Schrödinger equation with terms of cubic and quintic order in the wave function, namely

$$
\begin{aligned}
i \frac{\partial \Psi}{\partial t}= & -\frac{\partial^{2} \Psi}{\partial x^{2}}+v(x, t) \Psi+g_{3}(x, t)|\Psi|^{2} \Psi \\
& +g_{5}(x, t)|\Psi|^{4} \Psi
\end{aligned}
$$

whose function coefficients are written as

$v(x, t)=\omega(t) x^{2}+f_{1}(t) x+f_{2}(t)+\gamma^{2}(t) V[\gamma(t) x+\delta(t)]$,

$$
\begin{gathered}
g_{3}(x, t)=G_{3} \gamma(t) f[\gamma(t) x+\delta(t)], \\
g_{5}(x, t)=G_{5} h[\gamma(t) x+\delta(t)] .
\end{gathered}
$$

The reason for choosing the inhomogeneous coefficients in this way is going to be clarified below.

Now, we implement the following coordinate transformation and time rescaling [17]

$$
\begin{gathered}
x=\frac{\xi}{\bar{\gamma}(\tau)}-\frac{\bar{\delta}(\tau)}{\bar{\gamma}(\tau)}, \\
t-t_{0}=\int_{0}^{\tau} \frac{d \tau^{\prime}}{\bar{\gamma}^{2}\left(\tau^{\prime}\right)},
\end{gathered}
$$

with $\bar{\gamma}[\tau(t)]=\gamma(t)$ and $\bar{\delta}[\tau(t)]=\delta(t)$. Then, one can recast Eq. (5) as

$$
\begin{aligned}
i \bar{\gamma}\left(\bar{\gamma}_{\tau} \xi-\bar{\gamma}_{\tau} \bar{\delta}+\bar{\gamma} \bar{\delta}_{\tau}\right) \frac{\partial \bar{\Psi}}{\partial \xi}+i \bar{\gamma}^{2} \frac{\partial \bar{\Psi}}{\partial \tau} \\
=-\bar{\gamma}^{2} \frac{\partial^{2} \bar{\Psi}}{\partial \xi^{2}}+\bar{v}(\xi, \tau) \bar{\Psi}+G_{3} \bar{\gamma} \bar{f}(\xi)|\bar{\Psi}|^{2} \bar{\Psi} \\
\quad+G_{5} \bar{h}(\xi)|\bar{\Psi}|^{4} \bar{\Psi},
\end{aligned}
$$

where $\bar{\gamma}_{\tau}=d \bar{\gamma} / d \tau, \bar{\delta}_{\tau}=d \bar{\delta} / d \tau, \bar{\Psi}(\xi, \tau)=\Psi[x(\xi, \tau), t(\tau)]$, and

$$
\begin{aligned}
\bar{v}(\xi, \tau)= & \bar{\omega}(\tau) \frac{(\xi-\bar{\delta})^{2}}{\bar{\gamma}^{2}}+\bar{f}_{1}(\tau) \frac{(\xi-\bar{\delta})}{\bar{\gamma}} \\
& +\bar{f}_{2}(\tau)+\bar{\gamma}^{2}(\tau) V(\xi) .
\end{aligned}
$$

From the last two equations one can appreciate why we have chosen the specific dependence of $V, f$, and $h$ on $\xi=\gamma(t) x+$ $\delta(t)$. Equation (11) looks like a nonautonomous NLSE on $\xi$ and $\tau$, except for the first derivative term in the variable $\xi$. In order to eliminate that term, we redefine $\bar{\Psi}(\xi, \tau)$ as

$$
\bar{\Psi}=\sqrt{\bar{\gamma}(\tau)} \mathrm{e}^{-i \bar{\alpha}(\xi, \tau)} \psi(\xi, \tau),
$$

where $\bar{\alpha}(\xi, \tau)=\frac{\bar{\gamma}_{\tau}}{4 \bar{\gamma}} \xi^{2}+\left(\bar{\delta}_{\tau}-\frac{\bar{\gamma}_{\tau} \bar{\delta}}{\bar{\gamma}}\right) \frac{\xi}{2}-\bar{a}(\tau)$, with $\bar{a}(\tau)$ being an arbitrary function for the moment. By substituting Eq. (13) into Eq. (11) one gets

$$
\begin{aligned}
i \frac{\partial \psi}{\partial \tau}= & -\frac{\partial^{2} \psi}{\partial \xi^{2}}+U(\xi, \tau) \psi+G_{3} f(\xi)|\psi|^{2} \psi \\
& +G_{5} h(\xi)|\psi|^{4} \psi,
\end{aligned}
$$


where

$$
\begin{aligned}
U(\xi, \tau)= & \left(\bar{\omega}(\tau)-\frac{\bar{\gamma}^{3} \bar{\gamma}_{\tau \tau}}{4}\right) \frac{(\xi-\bar{\delta})^{2}}{\bar{\gamma}^{4}} \\
& +\left(\bar{f}_{1}(\tau)-\frac{\bar{\gamma}^{3} \bar{\delta}_{\tau \tau}}{2}\right) \frac{(\xi-\bar{\delta})}{\bar{\gamma}^{3}} \\
& +\frac{1}{\bar{\gamma}^{2}}\left(\bar{f}_{2}(\tau)+\frac{\bar{\delta}_{\tau}^{2} \bar{\gamma}^{2}}{4}+\bar{\gamma}^{2} \frac{d \bar{a}}{d \tau}\right)+V(\xi) .
\end{aligned}
$$

One can see why the factors involving $\gamma(t)$ are present in the expressions of $v(x, t)$ and $g_{3}(x, t)$. Moreover, one can also appreciate the contribution of the redefinition (13), specifically the contribution of the phase $\bar{\alpha}(\xi, \tau)$ to the redefinition of the trapping potential $U(\xi, \tau)$.

Now, one can choose $\gamma(\tau)$ and $\delta(\tau)$ such that

$$
\begin{aligned}
\bar{\omega}(\tau) & =\frac{\bar{\gamma}^{3} \bar{\gamma}_{\tau \tau}}{4}, \bar{f}_{1}(\tau)=\frac{\bar{\gamma}^{3} \bar{\delta}_{\tau \tau}}{2}, \\
\bar{f}_{2}(\tau) & =-\bar{\gamma}^{2}\left(\frac{\bar{\delta}_{\tau}^{2}}{4}+\frac{d \bar{a}}{d \tau}\right)
\end{aligned}
$$

and, by coming back to the original variables $(x, t)$, the functions $\omega, f_{1}$ and $f_{2}$ appear as

$$
\begin{aligned}
& \omega(t)=\frac{\gamma_{t t} \gamma-2 \gamma_{t}^{2}}{4 \gamma^{2}}, \quad f_{1}(t)=\frac{\delta_{t t} \gamma-2 \gamma_{t} \delta_{t}}{2 \gamma^{2}}, \\
& f_{2}(t)=-\frac{\delta_{t}^{2}}{4 \gamma^{2}}-\frac{d a}{d t},
\end{aligned}
$$

revealing the intrinsic connection between the frequency $\omega(t)$, the force $f_{1}(t)$, and the functions $\gamma(t)$ and $\delta(t)$. Note that $a(t)$ is an arbitrary function that could be chosen as $d a / d t=\left(\delta_{t} / 2 \gamma\right)^{2}$ if $f_{2}(t)$ were not present in Eq. (6). Thus, one removes the explicit time dependency of Eq. (14), that is,

$$
\begin{aligned}
i \frac{\partial \psi}{\partial \tau}= & -\frac{\partial^{2} \psi}{\partial \xi^{2}}+V(\xi) \psi+G_{3} f(\xi)|\psi|^{2} \psi \\
& +G_{5} h(\xi)|\psi|^{4} \psi,
\end{aligned}
$$

and the wave function (13) is written as

$$
\Psi(x, t)=\sqrt{\gamma(t)} \mathrm{e}^{-i \alpha(x, t)} \psi[\xi(x, t), \tau(t)],
$$

where $\alpha(x, t)=\frac{\gamma_{t}}{4 \gamma} x^{2}+\frac{\delta_{t}}{2 \gamma} x-a(t)$. We recall that the phase $\alpha(x, t)$ was introduced through the redefinition (13) and it contributes to the redefinition of the trapping potential, which could be eliminated thanks to the presence of the time-dependent driven harmonic oscillator terms [the three first terms $v(x, t)]$.

For stationary solutions in the variable $\tau$, that is, $\psi(\xi, \tau)=$ $\phi(\xi) \exp (-i E \tau)$, we have

$$
\frac{d^{2} \phi}{d \xi^{2}}=[V(\xi)-E] \phi+G_{3} f(\xi)|\phi|^{2} \phi+G_{5} h(\xi)|\phi|^{4} \phi .
$$

Since we still have a nonlinear equation with inhomogeneous nonlinearities, we are going to make further transformations in order to reach a nonlinear second-order differential equation with constant couplings. For that we redefine $\xi$ as a function of another variable $\zeta$, that is, $\xi=\bar{F}(\zeta)$, which leads us to the differential equation

$$
\begin{aligned}
\frac{d^{2} \phi}{d \zeta^{2}}-\frac{\bar{F}_{\zeta \zeta}}{\bar{F}_{\zeta}} \frac{d \phi}{d \zeta}= & \bar{F}_{\zeta}^{2}(V[\xi(\zeta)]-E) \phi \\
& +G_{3} \bar{F}_{\zeta}^{2} f[\xi(\zeta)]|\phi|^{2} \phi \\
& +G_{5} \bar{F}_{\zeta}^{2} h[\xi(\zeta)]|\phi|^{4} \phi,
\end{aligned}
$$

where $\bar{F}_{\zeta}=d \bar{F} / d \zeta$ and $\phi=\phi[\xi(\zeta)]$. By redefining the field as

$$
\phi(\zeta)=\sqrt{\bar{F}_{\zeta}} \Phi(\zeta)
$$

we reach Eq. (8) in terms of $\zeta$

$$
\frac{d^{2} \Phi}{d \zeta^{2}}=-\mu \Phi+G_{3}|\Phi|^{2} \Phi+G_{5}|\Phi|^{4} \Phi,
$$

where we have done the following identifications:

$$
\begin{aligned}
\mu & =-\bar{F}_{\zeta}^{2}(V[\xi(\zeta)]-E)+\frac{\bar{F}_{\zeta \zeta \zeta}}{2 \bar{F}_{\zeta}}-\frac{3 \bar{F}_{\zeta \zeta}^{2}}{4 \bar{F}_{\zeta}^{2}}, \\
f[\xi(\zeta)] & =\frac{1}{\bar{F}_{\zeta}^{3}}, \quad h[\xi(\zeta)]=\frac{1}{\bar{F}_{\zeta}^{4}} .
\end{aligned}
$$

As one can see, we have not defined the part of the trapping $V[\xi(\zeta)]$ yet. In fact, once we are going to work with a constant chemical potential, $V(\xi)$ is defined in terms of $\mu$ and $F(\xi)$. In terms of the variables $\xi$ and $\tau$ we have

$$
\begin{aligned}
& V(\xi)=\left(\frac{F^{\prime \prime}}{2 F^{\prime}}\right)^{2}-\left(\frac{F^{\prime \prime}}{2 F^{\prime}}\right)^{\prime}-\mu F^{2}+E, \\
& f(\xi)=F^{\prime^{3}}, \quad h(\xi)=F^{\prime^{4}},
\end{aligned}
$$

where $F^{\prime}=d F / d \xi$ and the field (21) becomes

$$
\phi(\xi)=\frac{1}{\sqrt{F^{\prime}(\xi)}} \Phi[\zeta(\xi)] .
$$

Finally, by returning to the original space-time coordinates $(x, t)$, the wave function can be obtained from Eqs. (18) and (25), that is,

$$
\Psi(x, t)=\frac{\sqrt{\gamma(t)}}{\sqrt{F^{\prime}[\xi(x, t)]}} \exp [-i \eta(x, t)] \Phi[F(\xi(x, t))],
$$

with $\eta(x, t)=\frac{\gamma_{t}}{4 \gamma} x^{2}+\frac{\delta_{t}}{2 \gamma} x-a(t)+E \int_{0}^{t} d t^{\prime} \gamma^{2}\left(t^{\prime}\right)$, where $a(t)$ is an arbitrary function.

Thus, we have shown, by means of transformation of variables, how the nonautonomous CQNLSE, Eq. (5), can be mapped onto a nonlinear, one-dimensional, second-order differential equation with cubic and quintic nonlinearities, Eq. (22). More than that, we have shown explicitly how the part of the trapping potential $V(\xi)$ and the nonlinearity functions $f(\xi)$ and $h(\xi)$ are related to the transformation function $F(\xi)=\bar{F}^{-1}(\xi)$ [see Eqs. (24)]. 


\section{THE MAPPING ONTO THE WEIERSTRASS FUNCTION}

Here we consider the cases in which $\Phi$ is a real function on $\zeta$ [Eq. (22)]. One can check that the solutions for Eq. (27) are also solutions of Eq. (22):

$$
\left(\frac{d \Phi}{d \zeta}\right)^{2}=\epsilon-\mu \Phi^{2}+\frac{G_{3}}{2} \Phi^{4}+\frac{G_{5}}{3} \Phi^{6},
$$

where $\epsilon$ is a real arbitrary constant. Moreover, we shown how to map such kind of equations [19] onto the nonlinear Weierstrass differential equation

$$
\left(\frac{d \wp}{d \zeta}\right)^{2}=4 \wp^{3}-g_{2} \wp-g_{3},
$$

where $g_{2}$ and $g_{3}$ are the Weierstrass invariants and the discriminant is $\Delta=g_{2}^{3}-27 g_{3}^{2}$. The values of the invariants and of the discriminant determine how the Weierstrass function are written in terms of the double-periodic Jacobi elliptic functions for $\Delta \neq 0$ [20], and the solutions for Eq. (27) can be included in one of the categories listed below:

(1) For $\epsilon \neq 0$, one has the following solution for $\Phi(\zeta)$ :

$$
\Phi(\zeta)=\sqrt{\frac{\epsilon}{\wp\left(\zeta, g_{2}, g_{3}\right)+\mu / 3}},
$$

where $\quad g_{2}=\frac{4}{3} \mu^{2}-2 G_{3} \epsilon$ and $g_{3}=\frac{8}{27} \mu^{3}-\frac{2}{3} G_{3} \mu \epsilon-$ $\frac{4}{3} G_{5} \epsilon^{2}$, which leads to the discriminant $\Delta=-\frac{4 \epsilon^{2}}{3}\left(6 \epsilon G_{3}^{3}+\right.$ $\left.36 \epsilon^{2} G_{5}^{2}+36 G_{3} G_{5} \epsilon \mu-3 \mu^{2} G_{3}^{2}-16 \mu^{3} G_{5}\right)$.

(2) For $\epsilon=0$ and $\mu \neq 0$ one sees that if $g_{2}=\frac{4}{3} \mu^{2}, g_{3}=$ $\frac{8}{27} \mu^{3}$, and $\Delta=0$, then the solution is

$$
\Phi(\zeta)=\left(\frac{G_{3}-2 \beta}{4 \mu}+\frac{\beta}{\wp\left(\zeta, g_{2}, g_{3}\right)+\mu / 3}\right)^{-1 / 2},
$$

where $\beta= \pm \sqrt{\frac{G_{3}^{2}}{4}+\frac{4}{3} \mu G_{5}}$.

(3) For $\epsilon=0$ and $\mu=0$ one gets $g_{2}=g_{3}=\Delta=0$ [in this case $\left.\wp(\zeta)=\zeta^{-2}\right]$ and

$$
\Phi(\zeta)=\left(-\frac{2 G_{5}}{3 G_{3}}+\frac{G_{3} \zeta^{2}}{2}\right)^{-1 / 2},
$$

All the examples with real-world applications considered in Refs. [13] and [14] may be reproduced and fit in one of these categories. We present next three additional examples which were not considered by them, which are solutions of Eq. (5).

\section{A. Examples}

Because the dark and bright solitons are of primary importance for developing concrete applications of BECs, many techniques are developed for manipulating and controlling the soliton's parameters and induce changes in their shapes which would be useful for applications. One possibility is to vary the atomic scattering length by means of external magnetic fields, that is, by using Feshbach resonances. People have been contemplating how to modulate the width and amplitude of a soliton in a controllable manner. It has been demonstrated that the variation of the scattering length provides a powerful tool for controlling the generation of bright and dark soliton trains; this technique can be used to modulate the bright soliton into very high local matter densities in both harmonic trap potential [21] and repulsive (inverted) harmonic trap potential [22].

We show by means of some examples that the width and the amplitude of the solitons can also be manipulated theoretically by focusing on the same form of the functions $F(\xi), \gamma(t)$, and $\delta(t)$ considered in Ref. [14] but with some different values of $\mu, G_{3}, G_{5}$, and $\epsilon$. Explicitly, we take $F(\xi)=\frac{\sqrt{3 \pi} b}{2 G_{3}^{1 / 3}} \operatorname{Erfi}\left(\frac{\xi}{\sqrt{3} b}\right)$ (this is the imaginary error function [20]), $\gamma(t)=\sqrt{\frac{2}{1+3 \cos ^{2}(2 t)}}$ and $\delta(t)=0$. We remark that this choice of $F(\xi)$, when substituted in $V(\xi)(24)$, implies into an extra contribution for the time-dependent frequency $\omega(t)$ of the driven harmonic oscillator, or in other words, the trapping potential $v(x, t)$ is a sole time-dependent driven harmonic oscillator for $\mu=0$.

\section{Example 1}

In real-world applications the nonlinearities and the confinement potential can be changed independently using an optical trap and magnetic-field-induced Feshbach resonance. In order to modulate the width of the dark soliton in a controllable manner, we gradually increase the self-interaction and simultaneously turn off the trapping potential.

Here, there is no way to turn off the ubiquitous trapping harmonic potential, but we can modulate the width of a dark soliton through a suitable choice of the chemical potential and the coefficient of the quartic self-interactions. Here, as in real-world applications, they are related to each other.

First we notice that Eq. (22) can be seen as the static equation of motion $d^{2} \Phi / d \zeta^{2}=d U / d \Phi$, where $U(\Phi)=$ $(1 / 2)\left(-\mu \Phi^{2}+\frac{G_{3}}{2} \Phi^{4}+\frac{G_{5}}{3} \Phi^{6}+c\right)$. If the parameters of the potential are chosen such that it presents only two minima, say at \pm 1 , and such that the chemical potential varies from positive to negative values (we notice that $d^{2} U / d \Phi_{\Phi=0}^{2}=-\mu$ ), the minimum energy solution $\Phi(\zeta)$, which connects the minima of $U(\Phi)$ at $\zeta \rightarrow \pm \infty$, is a kink which is deformable into a two-kink (double kink) as $\mu$ varies from positive to negative values.

Such $\Phi^{6}$ polynomial potentials have been used to study the phase transitions which come with domain wall splitting and the appearance of a wet phase in some ferroelectric [23] and paramagnetic [24] materials. The wetting transition can also take place in the deconfinement phase transition of SU(3) Yang-Mills theory [25], in supersymmetric QCD [26], and in thick brane world scenario [27,28].

We choose $\mu=2 a^{2}-1, \quad G_{3}=2\left(a^{2}-2\right), \quad G_{5}=3$, and $\epsilon=c=a^{2}$. The solution $\Phi(\zeta)$, for the chosen coefficients of $U(\Phi)$, falls into the first category of solutions shown in Eq. (29) with $g_{2}=\frac{4}{3}\left(1+a^{2}\right)^{2}$ and $g_{3}=-\frac{8}{27}\left(1+a^{2}\right)^{3}$ and $\wp\left(\zeta, g_{2}, g_{3}\right)=\frac{1}{3}\left(1+a^{2}\right)+(1+$ $\left.a^{2}\right) \operatorname{csch}^{2}\left(\sqrt{1+a^{2}} \zeta\right)$, such that we have

$$
\Phi(\zeta)=\frac{a \tanh \left(\sqrt{1+a^{2}} \zeta\right)}{\sqrt{\operatorname{sech}^{2}\left(\sqrt{1+a^{2}} \zeta\right)+a^{2}}} .
$$

The profiles of $|\Phi(\zeta)|^{2}$ for three different values of $\mu$ are shown in Fig. 1, from which one can see the increasing of the width of the soliton as $\mu$ becomes close to the critical value $\mu=-1$. 

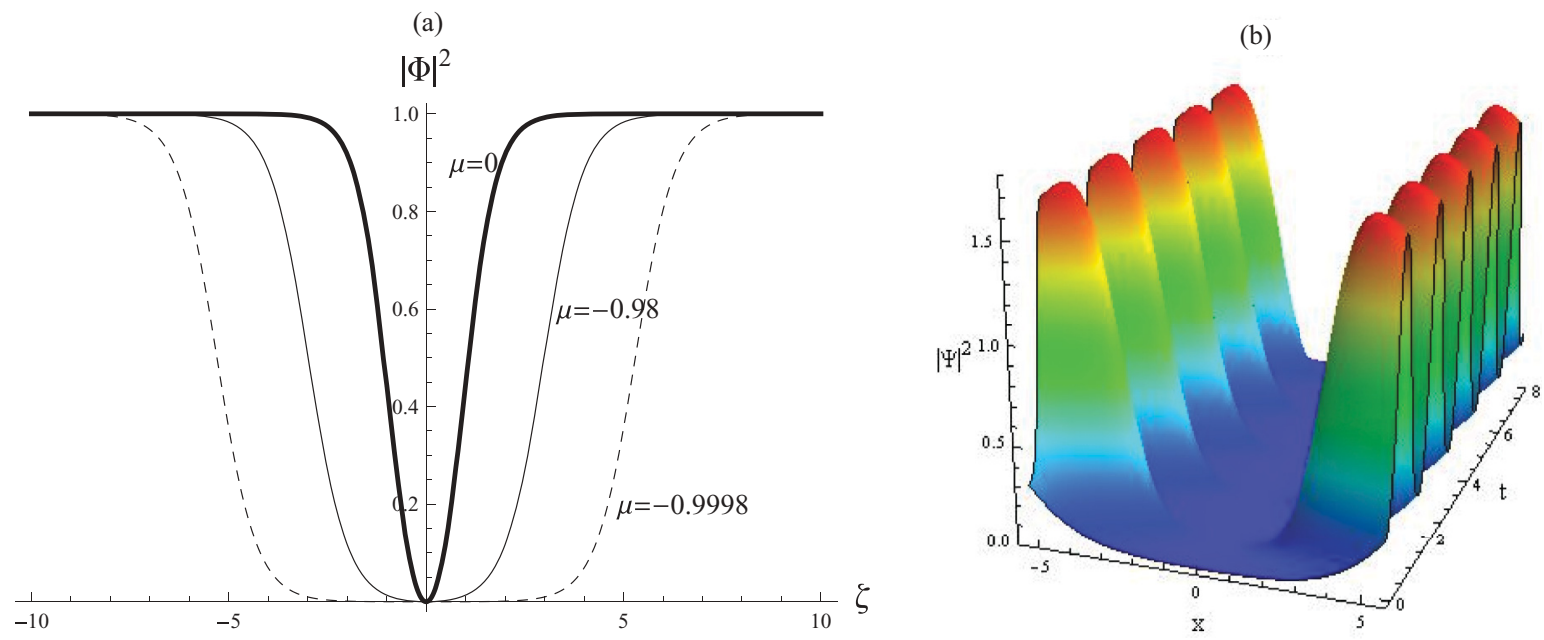

FIG. 1. (Color online) (a) Profiles of $|\Phi(\zeta)|^{2}$ from Eq. (32). (b) $|\Psi(x, t)|^{2}$ for the wide breathing dark soliton with $\lambda=0.001, b=10$, and $a=0.01$.

By using Eq. (26) we find the wave funcction

$$
\begin{aligned}
\Psi(x, t)= & G_{3}^{1 / 6} \sqrt{\gamma} \exp \left(-\frac{\gamma^{2} x^{2}}{6 b^{2}}\right) \exp [-i \eta(x, t)] \\
& \times \frac{a \sinh \left(\sqrt{1+a^{2}} \frac{\sqrt{3 \pi} b}{2 G_{3}^{1 / 3}} \operatorname{Erfi}\left(\frac{\gamma x}{\sqrt{3} b}\right)\right)}{\sqrt{1+a^{2} \cosh ^{2}\left(\sqrt{1+a^{2}} \frac{\sqrt{3 \pi} b}{2 G_{3}^{1 / 3}} \operatorname{Erfi}\left(\frac{\gamma x}{\sqrt{3} b}\right)\right)}},
\end{aligned}
$$

which is a wide breathing dark soliton for $a^{2}<1 / 2$.

\section{Example 2}

A bright soliton can also be modulated into a desired width and amplitude in a controllable manner by changing the scattering length and the trapping potential.

As in example 1, the soliton width can be modulated by a convenient choice of the parameters. Here we take values of $\mu$ less than the critical value $\mu=-1$ of the previous example and $U(\Phi)$ no longer positive, but with two symmetrically disposed global minima and one local minima at $\Phi=0$. We look for solutions such that $\Phi(\zeta \rightarrow \pm \infty) \rightarrow 0$. For that we take $\epsilon=c+k=0$, where $k$ is a constant of integration. From relations 18.12 .1 to 18.12 .3 of Ref. [20] one can see that $\wp\left(\zeta, 4 \mu^{2} / 3,8 \mu^{3} / 27\right)=(|\mu| / 3)\left(1+3 \operatorname{csch}^{2} \sqrt{|\mu| \zeta}\right)$ and by choosing $2 \beta / G_{3}=-\lambda^{2}<0$ (that is, $G_{5}<3 G_{3}^{2} / 16|\mu|$ ) and $G_{3}<0$, such that $\sqrt{-|\mu| / G_{3}}$ is real, we find that

$$
\Phi(\zeta)=\frac{2 \sqrt{-|\mu| / G_{3}}}{\sqrt{\lambda^{2} \cosh (2 \sqrt{|\mu|} \zeta)+1}} .
$$

We show profiles of $\Phi(\zeta)$ for three different values of $\lambda^{2}<$ 1 and $|\mu|=-G_{3}=4$ in Fig. 2. In the same figure we show also the amplitude $|\Psi(x, t)|^{2}$ for the wide breathing bright soliton.

Particularly, a very thin bright soliton can be obtained when $G_{5}=0$. We set $\mu=-1$ (the critical of $\mu$ ), $G_{3}=-1$, and $\epsilon=$ 0 , such that $g_{2}=\frac{4}{3}, g_{3}=-\frac{8}{27}, \Delta=0$, and $\Phi(\zeta)$ belongs to the second category of solutions listed in the previous section, namely

$$
\Phi(\zeta)=\left[\frac{1}{2}+\frac{1}{2}\left(\wp\left(\zeta, g_{2}, g_{3}\right)-\frac{1}{3}\right)^{-1}\right]^{-1 / 2}
$$

One can verify that $\wp\left(\zeta, g_{2}, g_{3}\right)=\frac{1}{3}+\operatorname{csch}^{2} \zeta$ and, consequently, $\Phi(\zeta)=\sqrt{2} \operatorname{sech} \zeta$

By using Eq. (26) we arrive at the breathing bright-soliton solution

$$
\begin{aligned}
\Psi(x, t)= & \mathrm{e}^{i \pi / 6} \sqrt{2 \gamma} \exp \left(-\frac{\gamma^{2} x^{2}}{6 b^{2}}\right) \\
& \times \exp [-i \eta(x, t)] \operatorname{sech}\left(-\frac{\sqrt{3 \pi} b}{2} \operatorname{Erfi}\left(\frac{\gamma x}{\sqrt{3} b}\right)\right),
\end{aligned}
$$

where $a(t)=\int\left[\gamma^{2}\left(E-\frac{1}{3 b^{2}}\right)\right] d t$.

\section{Example 3}

This is an interesting example because we can find three different analytic solutions with the same set of parameters, $\mu=5, G_{3}=10$, and $G_{5}=-3$. Among those solutions we find periodic solutions which were not presented in Ref. [14].

In the case $\epsilon=0$ and using Eq. (30) we find two very similar, periodic solutions:

$$
\begin{aligned}
& \Phi_{1}(\zeta)=\frac{\sqrt{10}}{\sqrt{(5-\sqrt{5})+2 \sqrt{5} \sin ^{2}(\sqrt{5} \zeta)}} \text { and } \\
& \Phi_{2}(\zeta)=\frac{\sqrt{10}}{\sqrt{(5+\sqrt{5})-2 \sqrt{5} \sin ^{2}(\sqrt{5} \zeta)}} .
\end{aligned}
$$



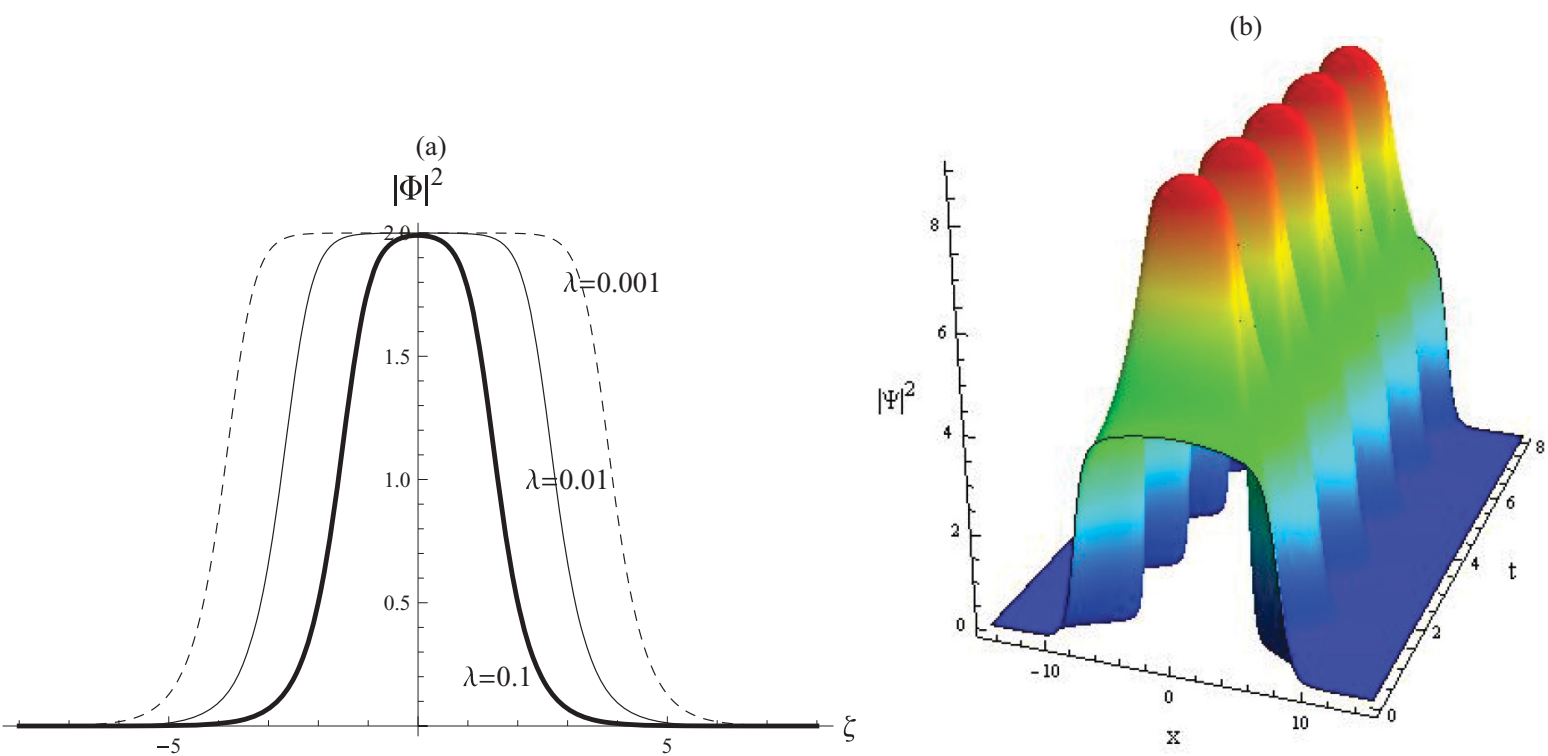

FIG. 2. (Color online) (a) Profiles of $|\Phi(\zeta)|^{2}$ from Eq. (34). (b) $|\Psi(x, t)|^{2}$ for the wide breathing bright soliton with $\lambda=0.001, b=10$.

However, if $\epsilon=\frac{5(4 \sqrt{10}-5)}{27}$ we find the kinklike configuration

$$
\begin{aligned}
\Phi(\zeta)= & \sqrt{\frac{4 \sqrt{10}-5}{3}} \\
& \times \frac{\sinh \left(\sqrt{\frac{5(\sqrt{10}-2)}{3}} \zeta\right)}{\sqrt{3(\sqrt{10}-2)+(\sqrt{10}+1) \sinh ^{2}\left(\sqrt{\frac{5(\sqrt{10}-2)}{3}} \zeta\right)}},
\end{aligned}
$$

which provides a breathing dark soliton solution for $\Psi(x, t)$.

\section{GENERALIZATIONS}

In this section we discuss some generalizations of the nonautonomous nonlinear Schrödinger equation. Particularly, we focus on two generalizations.

\section{A. Generalization of the trapping potential}

As shown in Eqs. (24), part of the potential is determined by a convenient choice of $F(\xi)$, such that $F^{\prime}(\xi)$ does not have zeros. Moreover, one can see that $V(\xi)$ has, for $\mu=E=0$, a structure similar to the one-dimensional supersymmetric quantum mechanics potential, with $F^{\prime \prime} / 2 F^{\prime}$ playing the role of supersymmetric superpotential. Then it would be interesting to analyze the influence that several trapping potentials in supersymmetric quantum mechanics could have while keeping the same nonlinearities on the nonautonomous nonlinear Schrödinger equation.

We have analyzed some of those possibilities and have found that the trigonometric Scarf and trigonometric RosenMorse potentials, which have in their spectrum only bound states (here we are using the same nomenclature for supersymmetric quantum mechanics potentials as that of Ref. [29]), are good candidates for the entrapment of breathing bright solitons in a spatially periodic trapping potential.
For the sake of brevity we just present the first case here; that is, we take

$$
F^{\prime}(\xi)=G_{3}^{-1 / 3} \sec ^{2 A / \alpha}(\alpha \xi),
$$

where $A, \alpha>0$ and $-\pi / 2 \leqslant \alpha \xi \leqslant \pi / 2$. Then $V(\xi)=A(A-$ $\alpha) \sec ^{2}(\alpha \xi)-A^{2}-\mu G_{3}^{-2 / 3} \sec ^{4 A / \alpha}(\alpha \xi) \quad$ and $g_{5}(x, t)=$ $G_{5} G_{3}^{-4 / 3} \sec ^{8 A / \alpha}(\alpha \xi)$.

It is always convenient to look for $\gamma(t)$ and $\delta(t)$ such that $\Psi(x, t)$ is well defined. We choose

$$
\gamma(t)=1+\left[1+\gamma_{1} \sin (t)+\gamma_{2} \sin (\sqrt{2} t)\right]^{2}, \quad \delta(t)=0 .
$$

We refer to Ref. [30] for more details on this function $\gamma(t)$. The time-dependent frequency and driven force are given by Eq. (16).

In order to get explicitly brightlike solution, let us consider $\mu=0, G_{3}=2, G_{5}=-3$, and $\epsilon=0$. The solution is given by

$$
\Psi(x, t)=\frac{2^{1 / 6} \gamma^{1 / 2} \exp [-i \eta(x, t)] \cos ^{A / \alpha}(\alpha \xi)}{\sqrt{1+F(\xi)^{2}}},
$$

whose modulus squared is shown in Fig. 3 for $A=\alpha=1$.

A breathing dark solution is obtained by setting $\mu=3$, $G_{3}=6, G_{5}=-3$, and $\epsilon=0$. It is given by

$$
\Psi(x, t)=\frac{6^{1 / 6} \gamma^{1 / 2} \exp [-i \eta(x, t)] F(\xi) \cos ^{A / \alpha}(\alpha \xi)}{\sqrt{1+F(\xi)^{2}}} .
$$

\section{B. Generalization of the nonlinearities}

We have shown in Eqs. (24) how the nonlinearities functions $g_{3}(x, t)$ and $g_{5}(x, t)$ [see Eqs. (5)-(8)] must be intrinsically related to each other and given in terms of the functions $\gamma(t)$ and $F^{\prime}(\xi)$, such that the mapping of Eq. (5) onto Eq. (22) could be realized. From a close inspection of those relations we have found that the approach used here can be used to map a nonautonomous Schrödinger equation with nonpolynomial nonlinearity onto a static sine-Gordon-like 


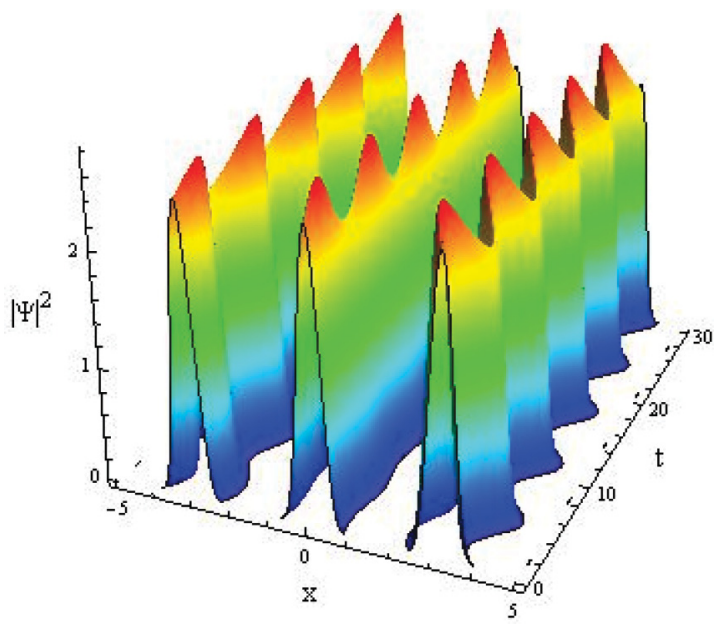

FIG. 3. (Color online) $|\Psi(x, t)|^{2}$ for the bright soliton from Eq. (41) with $\gamma_{1}=0, \gamma_{2}=0.1$. The periodic structure is due to the periodicity of the circular trapping potential.

equation. The first paper in Ref. [30] proposed a polynomial nonlinearity up to $N$ th order in $\Psi(x, t)$, with functional coefficients related to each order such that the nonautonomous NLSE could be mapped onto a stationary NLSE with constant coefficients. Here we present inverse transformations to obtain a nonautonomous NLSE with nonpolynomial nonlinearity from a stationary NLSE with nonpolynomial nonlinearity. Particularly, we consider the sine-Gordon equation, which is satisfied by $\Phi(\zeta)$, namely

$$
\frac{d^{2} \Phi(\zeta)}{d \zeta^{2}}=\frac{1}{b^{2}} \sin [b \Phi(\zeta)]=\sum_{n=0}^{\infty} \frac{(-1)^{n}}{(2 n+1) !} b^{2 n-1} \Phi^{2 n+1},
$$

where $b$ is positive constant. By using the transformation $\zeta=$ $F(\xi)$ and the redefinition of $\Phi[F(\xi)]=\sqrt{F^{\prime}(\xi)} \phi(\xi)$ we find that

$$
\begin{aligned}
\frac{d^{2} \phi}{d \xi^{2}}= & {\left[\left(\frac{F^{\prime \prime}}{2 F^{\prime}}\right)^{2}-\left(\frac{F^{\prime \prime}}{2 F^{\prime}}\right)^{\prime}\right] \phi } \\
& +\sum_{n=0}^{\infty} \frac{(-1)^{n}}{(2 n+1) !}\left(F^{\prime}\right)^{n+2} b^{2 n-1} \phi^{2 n+1} .
\end{aligned}
$$

Now, by considering that $\phi(\xi)=\psi(\xi, \tau) e^{i E \tau}$, we find that $\Psi(\xi, \tau)$ satisfies the nonautonomous NLSE

$$
\begin{aligned}
i \frac{\partial \psi}{\partial \tau}= & -\frac{\partial^{2} \psi}{\partial \xi^{2}}+V(\xi) \psi \\
& +\sum_{n=0}^{\infty} \frac{(-1)^{n}}{(2 n+1) !}\left(F^{\prime}\right)^{n+2} b^{2 n-1}|\psi|^{2 n} \psi,
\end{aligned}
$$

where $V(\xi)=\left(\frac{F^{\prime \prime}}{2 F^{\prime}}\right)^{2}-\frac{d}{d \xi}\left(\frac{F^{\prime \prime}}{2 F^{\prime}}\right)+E$.

Now, the inverse of transformations in Eqs. (9) and (10), namely,

$$
\xi=\gamma(t) x+\delta(t) \quad \text { and } \quad \tau-\tau_{0}=\int_{0}^{t} d t^{\prime} \gamma^{2}\left(t^{\prime}\right),
$$

lead us to

$$
\begin{aligned}
\frac{i}{\gamma^{2}} \frac{\partial \psi}{\partial \tau}= & \frac{i}{\gamma^{3}}\left(\gamma_{t} x+\delta_{t}\right) \frac{\partial \psi}{\partial x}-\frac{1}{\gamma^{2}} \frac{\partial^{2} \psi}{\partial x^{2}}+V[\xi(x, t)] \psi \\
& +\sum_{n=0}^{\infty} \frac{(-1)^{n}}{(2 n+1) !} b^{2 n-1}\left(F^{\prime}\right)^{n+2}|\psi|^{2 n} \psi
\end{aligned}
$$

where $\psi=\psi[\xi(x, t), \tau(t)]$. The first derivative in $x$ can be eliminated by the following redefinition of the wave function $\psi(x, t)=e^{i \alpha(x, t)} \Psi(x, t) / \sqrt{\gamma(t)}$, where $\alpha(x, t)=\frac{\gamma_{t}}{4 \gamma} x^{2}+$ $\frac{\delta_{t}}{2 \gamma} x-a(t)$. Then we finally obtain the nonautonomous NLSE with a generalized nonlinearity

$$
\begin{aligned}
i \frac{\partial \Psi}{\partial t}= & -\frac{\partial^{2} \Psi}{\partial x^{2}}+v(x, t) \Psi \\
& +\sum_{n=0}^{\infty} \frac{(-1)^{n}}{(2 n+1) !} b^{2 n-1}\left(\frac{F^{\prime}}{\gamma}\right)^{n+2}|\Psi|^{2 n} \Psi \\
= & \frac{\delta \mathbf{H}}{\delta \Psi^{*}(x, t)},
\end{aligned}
$$

where

$$
\begin{aligned}
v(x, t)= & \frac{\gamma_{t t} \gamma-2 \gamma_{t}^{2}}{4 \gamma^{2}} x^{2}+\frac{\delta_{t t} \gamma-2 \gamma_{t} \delta_{t}}{2 \gamma^{2}} x \\
& -\frac{\delta_{t}^{2}}{4 \gamma^{2}}-\frac{d a}{d t}+\gamma^{2} V[\xi(x, t)]
\end{aligned}
$$

and

$$
\begin{aligned}
\mathbf{H}= & \int d z\left\{\Psi^{*}(z, t)\left[-\frac{\partial^{2}}{\partial z^{2}}+v(z, t)\right] \Psi(z, t)\right. \\
& \left.+\frac{2}{b^{3}} \gamma^{3} F^{\prime}[\xi(z, t)] \cos \left(b \sqrt{\frac{F^{\prime}[\xi(z, t)]}{\gamma}}|\Psi|\right)\right\} .
\end{aligned}
$$

In this specific generalization the soliton amplitude is periodic and is given by

$$
|\Psi(x, t)|^{2}=\frac{4 \gamma(t)\{\arccos [v \operatorname{sn}(F(\xi) / \sqrt{b} \mid v)]\}^{2}}{b^{2} F^{\prime}(\xi)},
$$

where $\operatorname{sn}(z \mid v)$ is the snoidal Jacobi elliptic function with elliptic parameter $0 \leqslant v \leqslant 1$. For $v=1, \operatorname{sn}(z \mid v)=\tanh (z)$ and we have a breathing dark soliton.

\section{CONCLUSIONS}

In this work we have shown that an approach used to tackle the Schrödinger equation with explicit time-dependent potential parameters can be extended to the case of a nonautonomous NLSE. As a consequence we were able to reproduce results originally obtained through the use of an ansatz together with similarity transformations $[13,14]$. Besides, this procedure leads us to interesting solutions of that problem and particularly some configurations of wide soliton solutions.

Furthermore, we extended the approach in order to deal with systems with generalized nonlinearities and trapping potentials which are a mixing of the (driven) time-dependent harmonic oscillator and circular functions. 
We believe that the approach applied here can be used to deal with the cases of damped-driven nonlinear Schrödinger equation with time-dependent cubic and quintic nonlinearities, such as the one treated originally in Ref. [12].

Moreover, extensions of this work for the cases with two or more fields are under development.

\section{ACKNOWLEDGMENTS}

L.E.A.M. thanks the Brazilian funding agency CAPES for support through a scholarship under the PEC-PG program. This work is also partially supported by CNPq (Projects No. 482043/2011-3, No. 304352/2009-8, and No. 304252/2011-5).
[1] C. Sulem, and P.-L. Sulem, The Nonlinear Schrödinger Equation: Self-focusing and Wave Collapse (Springer, New York, 1999).

[2] F. Dalfovo, S. Giorgini, L. P. Piataevskii, and S. Stringari, Rev. Mod. Phys. 71, 463 (1999).

[3] A. Das, Integrable Models (World Scientific, Singapore, 1989).

[4] D. Mihalache, D. Mazilu, M. Bertolotti, and C. Sibilia, J. Opt. Soc. Am. B 5, 565 (1988); J. Herrmann, Opt. Commun. 87, 161 (1992); Y. J. Chen, Phys. Rev. E 55, 1221 (1997).

[5] Yu. S. Kivshar and G. P. Agrawal, Optical Solitons: From Fibers to Photonic Crystals (Academic Press, Boston, 2003).

[6] A. Grifin, D. W. Snoke, and S. Stringari (eds.), Bose Einstein Condensation (Cambridge University Press, Cambridge, 1996); L. Pitaevskii and S. Stringari, Bose-Einstein Condensation (Clarendon Press, New York, 2003); S. Inouye et al., Nature (London) 392, 151 (1998); F. Kh. Abdullaev, A. Gammal, L. Tomio, and T. Frederico, Phys. Rev. A 63, 043604 (2001); E. Braaten and H. W. Hammer, Phys. Rev. Lett. 87, 160407 (2001); M. Theis et al., ibid. 93, 123001 (2004).

[7] S. Burger, K. Bongs, S. Dettmer, W. Ertmer, and K. Sengstock, Phys. Rev. Lett. 83, 5198 (1999); J. Denshlag et al., Science 287, 97 (2000)

[8] M. I. Rodas-Verde, H. Michinel, and V. M. Pérez-García, Phys. Rev. Lett. 95, 153903 (2005); J. Belmont-Beitia et al., ibid. 98, 064102 (2007); Y. V. Kartashov, B. A. Malomed, and L. Torner, Rev. Mod. Phys. 83, 247 (2011).

[9] F. Kh. Abullaev et al., Phys. Rev. Lett. 90, 230402 (2003); V. M. Pérez-García, V. V. Konotop, and V. A. Brazhanyi, ibid. 92, 220403 (2004); H. Saito and M. Ueda, Phys. Rev. Lett. 90, 040403 (2003); V. V. Konotop and P. Pacciani, ibid. 94, 240405 (2005).

[10] B. A. Malomed, Soliton Management in Periodic Systems (Springer, New York, 2006).

[11] V. N. Serkin, A. Hasegawa, and T. L. Belyaeva, Phys. Rev. Lett. 98, 074102 (2007); J. Mod. Opt. 57, 1456 (2010).

[12] V. N. Serkin and A. Hasegawa, Phys. Rev. Lett. 85, 4502 (2000); V. N. Serkin, T. L. Belyaeva, I. V. Alexandrov, and G. M. Melchor, Proc. SPIE 4271, 292 (2001).
[13] J. Belmonte-Bietia, V. M. Pérez-García, V. Vekslerchik, and V. V. Konotop, Phys. Rev. Lett. 100, 164102 (2008).

[14] A. T. Avelar, D. Bazeia, and W. B. Cardoso, Phys. Rev. E 79, 025602(R) (2009).

[15] R. K. Colegrave and A. Vahabpour-Roudsari, Opt. Acta 32, 495 (1985), and references therein.

[16] Y. Ben-Aryeh and A. Mann, Phys. Rev. A 32, 552 (1985); Phys. Rev. Lett. 54, 1020 (1985).

[17] C. Farina de Souza and A. de Souza Dutra, Phys. Lett. A 123, 297 (1987).

[18] R. Jackiw, Ann. Phys. (NY) 129, 183 (1980).

[19] A. de Souza Dutra, M. Hott, and F. F. Bellotti, arXiv:1002.4609v1.

[20] M. Abramowitz and I. Stegun, Handbook of Mathematical Functions (Dover, Toronto, 1965).

[21] F. Kh. Abdullaev and M. Salerno, J. Phys. B 36, 2851 (2003).

[22] Z. X. Liang, Z. D. Zhang, and W. M. Liu, Phys. Rev. Lett. 94, 050402 (2005).

[23] S. Dorfman, D. Fuks, A. Gordon, and E. Kotomin, Thin Solid Films 336, 149 (1998).

[24] A. Gordon, T. Salditt, I. D. Vagner, and P. Wyder, Phys. Rev. B 43, 3775 (1991).

[25] Z. Frei and A. Patkós, Phys. Lett. B 229, 102 (1989); T. Trappenberg and U.-J. Wiese, Nucl. Phys. B 372, 703 (1992).

[26] A. Campos, K. Holland, and U.-J. Wiese, Phys. Rev. Lett. 81, 2420 (1998)

[27] A. Campos, Phys. Rev. Lett. 88, 141602 (2002).

[28] A. E. R. Chumbes and M. B. Hott, Phys. Rev. D 81, 045008 (2010); A. E. R. Chumbes, A. E. O. Vasquez, and M. B. Hott, ibid. 83, 105010 (2011).

[29] F. Cooper, A. Khare, and U. Sukhatme, Phys. Rep. 251, 267 (1995)

[30] W. B. Cardoso, A. T. Avelar, and D. Bazeia, Nonlinear Anal. Real World Appl. 11, 4269 (2010); S. Peil, J. V. Porto, B. Laburthe Tolra, J. M. Obrecht, B. E. King, M. Subbotin, S. L. Rolston, and W. D. Phillips, Phys. Rev. A 67, 051603(R) (2003). 